\title{
Dynamic and Opposing Adjustment of Movement Cancellation and Generation in an Oculomotor Countermanding Task
}

\author{
Brian D. Corneil, ${ }^{1,2,3}$ Joshua C. Cheng, ${ }^{1}$ and Samanthi C. Goonetilleke ${ }^{1}$ \\ Departments of ${ }^{1}$ Physiology and Pharmacology and ${ }^{2}$ Psychology, Western University, London, Ontario N6A 5C1, Canada and ${ }^{3}$ The Brain and Mind \\ Institute, Robarts Research Institute, London, Ontario N6A 5K8, Canada
}

\begin{abstract}
Adaptive adjustments of strategies help optimize behavior in a dynamic and uncertain world. Previous studies in the countermanding (or stop-signal) paradigm have detailed how reaction times (RTs) change with trial sequence, demonstrating adaptive control of movement generation. Comparatively little is known about the adaptive control of movement cancellation in the countermanding task, mainly because movement cancellation implies the absence of an outcome and estimates of movement cancellation require hundreds of trials. Here, we exploit a within-trial proxy of movement cancellation based on recordings of neck muscle activity while human subjects attempted to cancel large eye-head gaze shifts. On a subset of successfully cancelled trials where gaze remains stable, small head-only movements to the target are actively braked by a pulse of antagonist neck muscle activity. The timing of such antagonist muscle recruitment relative to the stop signal, termed the "antagonist latency," tended to decrease or increase after trials with or without a stop-signal, respectively. Over multiple time scales, fluctuations in the antagonist latency tended to be the mirror opposite of those occurring contemporaneously with RTs. These results provide new insights into the adaptive control of movement cancellation at an unprecedented resolution, suggesting it can be as prone to dynamic adjustment as movement generation. Adaptive control in the countermanding task appears to be governed by a dynamic balance between movement cancellation and generation: shifting the balance in favor of movement cancellation slows movement generation, whereas shifting the balance in favor of movement generation slows movement cancellation.
\end{abstract}

\section{Introduction}

A central aspect to executive control is the ability to adjust one's strategy to optimize behavior. Such adaptive control can be exerted over a variety of time scales, reflecting events in the immediate past. For example, in the countermanding (or stop-signal) paradigm, which pits movement generation against movement cancellation by occasionally requiring subjects to try to cancel an impending response upon presentation of an infrequent stop signal (Logan and Cowan, 1984), saccadic reaction times (RTs) are longer or shorter after successive trials with or without a stop signal, respectively (Cabel et al., 2000; Kornylo et al., 2003; Ozyurt et al., 2003; Emeric et al., 2007; Nelson et al., 2010). The sensitivity of movement generation processes to immediate trial

Received May 26, 2012; revised April 8, 2013; accepted May 8, 2013.

Author contributions: B.D.C., J.C.C., and S.C.G. designed research; B.D.C., J.C.C., and S.C.G. performed research; B.D.C., J.C.C., and S.C.G. analyzed data; B.D.C. wrote the paper.

This work was supported by operating grants from the Canadian Institutes of Health Research (MOP\#93796) and a Discovery Grant from the Natural Sciences and Engineering Research Council of Canada. We thank Drs. David Irwin and Pieter Medendorp for comments on an earlier version of this manuscript, and Dr. Frederick Verbruggen for helpful discussions. We also thank Jeffrey Wong for his contribution to data collection and analysis, Kevin Barker for technical assistance, and Dr. Timothy Doherty for his expertise with intramuscular insertions.

The authors declare no competing financial interests.

Correspondence should be addressed to Brian D. Corneil, The Brain and Mind Institute, Robarts Research Institute, London, 0N N6A 5K8, Canada. E-mail: bcorneil@uwo.ca.

DOI:10.1523/JNEUROSCI.2543-12.2013

Copyright $\odot 2013$ the authors $\quad 0270-6474 / 13 / 339975-10 \$ 15.00 / 0$ history may reflect adaptive control of its priority (Bissett and Logan, 2011).

Far less is known about the adaptive control of movement cancellation in this task, particularly at short time scales. Although the countermanding paradigm permits estimation of the stop-signal reaction time (SSRT), or the time required for movement cancellation, this estimate requires analysis of hundreds of trials. Furthermore, the rapid modification in movement generation with recent trial history itself influences the probability of responding on stop-signal trials. Following a stop trial for example, do subjects selectively slow movement generation without influencing movement cancellation, slow both movement generation and movement cancellation simultaneously, or slow movement generation while simultaneously expediting movement cancellation? Similar questions can be asked about fluctuations in movement cancellation over longer time scales, as subjects perhaps subtly modify their strategy or simply become fatigued. Answers to these questions would advance our understanding of movement cancellation and executive control, but the SSRT estimate simply does not have the required resolution.

Our recent work has described a neuromuscular proxy for the cancellation of an oculomotor program available on a single-trial basis. When attempting to cancel an impending eye-head gaze shift, human subjects occasionally generate a small head-only movement toward the target even though the gaze shift is cancelled successfully (Corneil and Elsley, 2005). Recordings of neck 
muscle activity demonstrate that this small head movement is actively braked by a pulse of antagonist neck muscle activity (Goonetilleke et al., 2010). The timing of such activity relative to the stop signal, which we term the antagonist latency (see Fig. $1 A)$, correlates positively with the SSRT across multiple subjects (Goonetilleke et al., 2010), as does the within-subject change in both SSRT and antagonist latency across different stop-signal intensities (Goonetilleke et al., 2012). Here, we report fluctuations in antagonist latency across a variety of time scales and compare such fluctuations to those occurring contemporaneously with the RT of the saccadic gaze shift. Our results reveal opposing trends in all of these measures, including with immediate trial history. To our knowledge, these results constitute the first direct evidence that movement cancellation can be as prone to dynamic adjustment as movement generation, and suggest that adaptive control in this task is regulated through a balance that can expedite movement cancellation at the cost of movement generation and vice versa.

\section{Materials and Methods}

The experimental infrastructure and behavioral task is very similar to that described in our previous work (Goonetilleke et al., 2010, 2012). Eighteen different human subjects (mean age, 27; four female) participated in experimental procedures approved by the University Research Ethics Board for Health Science Research at the University of Western Ontario and were in accordance with the 1967 Declaration of Helsinki. Subjects gave informed consent and were aware they could terminate testing at any time.

The data set analyzed here is pooled across three experiments. As described in more detail below, one difference in these experiments is the intensity of the visual stop signal, which was either bright (Experiments 1 or 3) or dim or bright (Experiment 2). Data from Experiment 1 were collected from eight subjects. This data set was used in our initial report (Goonetilleke et al., 2010) describing neck muscle recruitment during the countermanding of eye-head gaze shifts, and is reexamined here for the effects of time and trial sequence. Data for Experiment 2 were collected from eight subjects. This data set was used in a different manuscript (Goonetilleke et al., 2012) to examine the effects of stop-signal intensity on the SSRT and the antagonist latency, and is also reexamined here for the effects of time and trial sequence. Finally, we also collected data from an additional 10 subjects for Experiment 3, using the same procedures as in Experiment 2 with the exception of only using a bright stop signal. This data set was collected solely for this manuscript. Therefore, the data set examined here encompasses a total of 26 different experimental sessions, across 18 different subjects (two subjects performed in all three experiments, two subjects performed in Experiments 1 and 2, and four subjects performed in Experiments 2 and 3).

Behavioral task. Subjects performed an oculomotor countermanding task with their heads unrestrained. On no-stop trials, subjects generated coordinated eye-head gaze shifts from a central fixation point (FP) to a visual target presented either $60^{\circ}$ left or $60^{\circ}$ right. All visual stimuli were LEDs. A $200 \mathrm{~ms}$ gap was introduced between FP disappearance and visual target presentation in Experiment 1, and FP disappearance and visual target presentation was synchronous in Experiments 2 and 3. On a subset of trials ( $\sim 30 \%$, termed stop trials), a visual stop signal was presented. The visual stop signal was either the reappearance of the central FP (Experiment 1) or a visual stimulus presented directly above the central FP (Experiments 2 and 3). In Experiment 2, the stop signal was either bright or dim, with equal probability. In Experiments 1 and 3, the stop signal was always bright. The interval between target presentation and the visual stop signal is termed the stop-signal delay (SSD; Fig. $1 \mathrm{~A}$ ). In Experiment 1 , the SSD was selected pseudorandomly from a set of six SSDs that spanned $200 \mathrm{~ms}$ to cover the minimum to maximum of the inhibition function that describes movement probability as a function of SSD. In Experiments 2 and 3, the SSD was varied adaptively using a staircasing algorithm so that subjects successfully canceled gaze shifts on $\sim 50 \%$ of all stop trials, with the SSD increasing (decreasing) by 40 or $80 \mathrm{~ms}$ with equal probability if the gaze shift on the previous stop trial was successfully (unsuccessfully) cancelled. In Experiment 2, independent staircasing algorithms were run for bright or dim stop signals, with both initialized to $80 \mathrm{~ms}$.

Subjects were instructed only to try to withhold a gaze shift upon presentation of the stop signal, and were not given any explicit instructions about eye-head coordination or performance feedback. Subjects in Experiment 1 completed three blocks of 200 trials each (600 trials per subject total). Subjects in Experiments 2 and 3 completed six blocks of 204 trials each (1224 trials per subject total). The selection of target direction (left or right), trial type (stop or no-stop trials), SSD (Experiment 1), or stop-signal intensity (Experiment 2) on a given trial was pseudorandomized by customized LabView (National Instruments) programs so that all conditions were presented an appropriate number of times within a block. Short breaks were introduced between blocks.

Data collection and analysis. We recorded horizontal eye and head movements (summed together off-line to yield gaze in space), and electromyographic (EMG) activity from splenius capitis (SPL). SPL recordings were made bilaterally via intramuscular electrodes using staggered monopolar insertions to characterize SPL recruitment across multiple motor units. Off-line analyses included a trial-by-trial inspection and classification of all movement and EMG traces conducted in a graphical user interface.

Our previous work (Goonetilleke et al., 2012) demonstrated that antagonist latencies are shorter with a brighter stop signal. To ensure that this difference does not confound the results from Experiment 2, our analysis of how the antagonist latency or SSRT changes within or across blocks of trial examined only stop trials with a bright stop signal, or were conducted separately for dim and bright stop signals. Our subsequent triplet analysis of changes in antagonist latency across sequences of three trials only used data where the stop-signal intensity was the same on trials $n-1$ and $n+1$.

\section{Results}

All subjects generated one of three movement sequences on a given stop trial: they either generated eye-head gaze shifts toward the target (noncanceled trials), fully canceled motion of the eyes or head (cancelled trials), or generated a head-only movement toward the target that was compensated for by eye-in-head motion that maintained gaze stability (Fig. $1 A$ ). Our previous work has documented the frequency of these three movement sequences, how their propensity changes across SSD, and the profile of bilateral neck muscle recruitment during each sequence (Corneil and Elsley, 2005; Goonetilleke et al., 2010, 2012). Headonly errors are uniquely associated with a burst of neck EMG activity on the antagonist muscle (Fig. $1 A$ ). Because such antagonist recruitment occurs during the outbound head movement, it is a lengthening contraction that serves to actively brake the head.

\section{The antagonist latency extracted from head-only movements is a proxy for the time of movement cancellation}

Head-only movements are examples of successfully canceled gaze shifts. During this movement sequence, head movements are first initiated by agonist neck muscle activity and then actively braked by antagonist neck muscle recruitment. Our central hypothesis is that the antagonist latency relative to the SSD is a proxy of oculomotor cancellation that can be extracted from head-only movement trials. Consistent with this, antagonist muscle activity is not seen on no-stop trials, on fully cancelled trials, or on noncancelled trials where gaze attains the peripheral target. Antagonist muscle recruitment is observed during noncancelled gaze shifts that do not attain the target (Goonetilleke et al., 2010). Large eye-head gaze shifts can be truncated in midflight in this and related tasks (Corneil et al., 1999; Corneil and Elsley, 2005), suggesting that processing of the stop signal continues during move- 

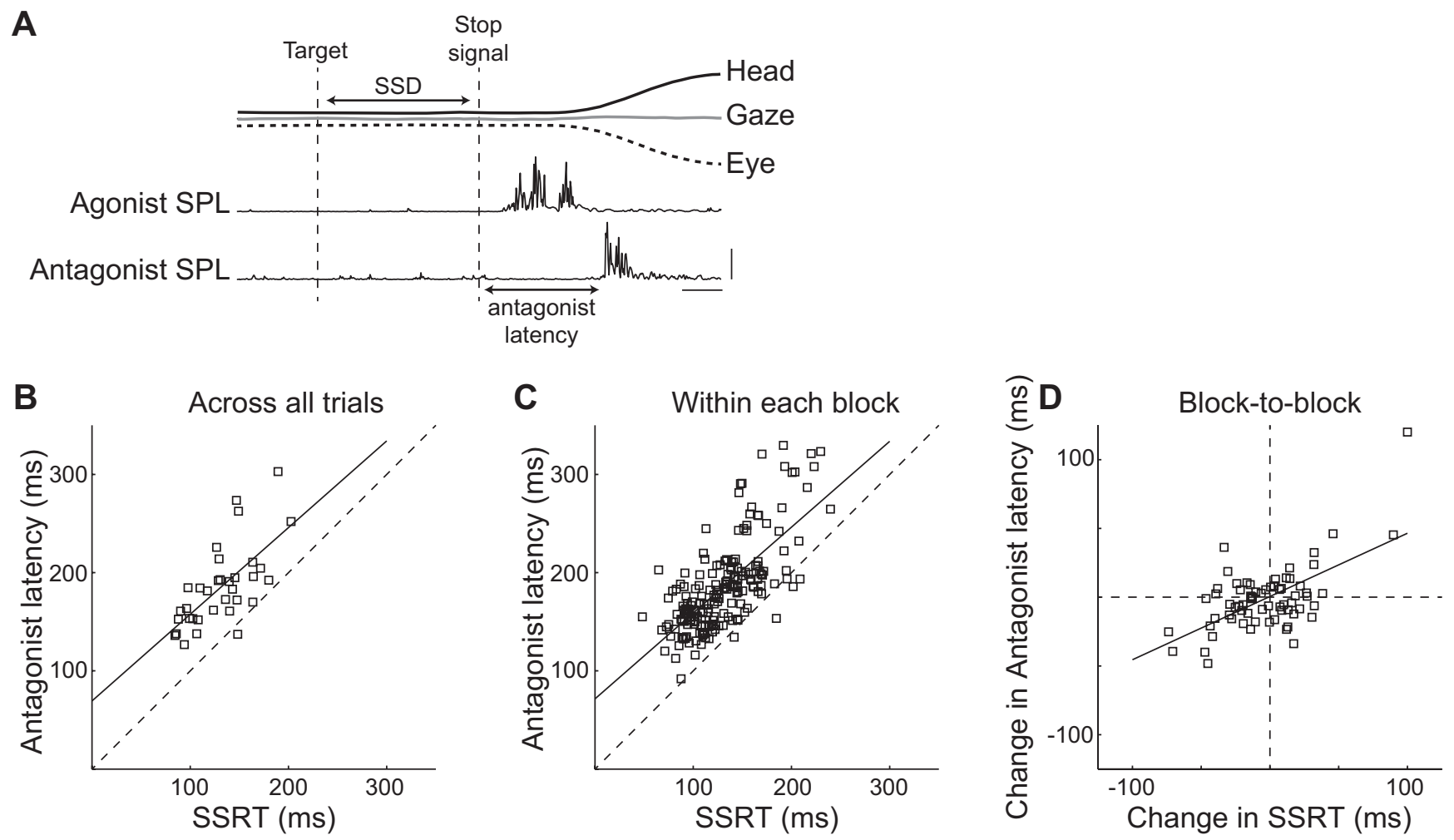

Figure 1. A, Depiction of a head-only error on a stop trial. The SSD is the time between the presentation of the target and stop signal. During a head-only error, gaze stability is maintained by compensatory eye motion within the head. The antagonist latency is the time from stop-signal presentation to the onset of recruitment of the antagonist muscle that brakes the ongoing head movement toward the target. SPL is an ipsilateral head turning neck muscle. Calibration: $50 \mathrm{~ms} ; 10^{\circ}$ for movement traces, $30 \mu V$ for EMG activity. $\boldsymbol{B}$, C, Comparison of SSRT estimates and average antagonist latency, calculated either across all trials $(\boldsymbol{B})$ or with each block of $\sim 200$ trials $(\boldsymbol{C})$; note the positive correlations in each case. Squares in $\boldsymbol{B}$ represent different subjects; squares in $\boldsymbol{C}$ represent different blocks of $\sim 200$ trials. Solid diagonal lines show a linear regression ( $p<10^{-4}$ for both). $\boldsymbol{D}$, Comparison of block-to-block changes in SSRT and the antagonist latency, showing data only from Experiments 2 and 3. Dashed lines divide quadrants, and solid line shows a linear regression $\left(p<10^{-8}\right)$.

ment generation. In this and our previous countermanding work, we analyze antagonist muscle recruitment only during head-only errors, rather than truncated noncancelled gaze shifts, to avoid confounds that may be associated with gaze shift initiation.

Across our sample, subjects generated between 13 and 55 head-only movements in Experiment 1 (mean \pm SD, $37 \pm 12$ ), between 88 and 166 head-only movements in Experiment 2 (mean $\pm \mathrm{SD}, 128 \pm 30$ ), and between 41 and 172 head-only movements in Experiment 3 (mean \pm SD, $132 \pm 38$; recall 600 trials were collected in Experiment 1 and 1224 trials in Experiments 2 and 3). There was no difference in the propensity to generate head-only movements in Experiments 2 and 3 (two-way $t$ test, $t_{(16)}=0.23, p=0.82$ ), but head-only movements were significantly less likely on a percentage basis in Experiment1 compared to Experiments 2 and 3 (two-way $t$ test, $t_{(24)}=3.7$, $p=0.001)$. This difference relates to the use of the staircasing algorithm in Experiments 2 and 3, which tends to sample intermediate SSDs at which head-only movements are more likely (Corneil and Elsley, 2005). Regardless, we obtained a considerable yield of head-only movements from all experiments.

Our previous work has shown that the timing of this antagonist muscle recruitment relative to the stop signal, which we term the "antagonist latency," correlates positively with the SSRT and decreases for longer SSDs as predicted by the race model, since longer SSDs provide less time for stopping (Goonetilleke et al., 2010, 2012). These findings were replicated in Experiment 3. Furthermore, both the SSRT and antagonist latency are shorter for brighter stop signals, and our recent paper showed that these intensity-dependent changes in SSRT and antagonist latency within a given subject are correlated as well (Goonetilleke et al., 2012). The relationship between the average antagonist latency and SSRT across all trials (calculated via the average of the mean and integration methods) (for more details, see Goonetilleke et al., 2010) is shown in Figure $1 B\left(r=0.67, p<10^{-4}\right.$ for the regression in Fig. $1 B$ ).

While this result replicates previous findings, we sought to examine how the relationship between the SSRT and the antagonist latency changes through time. To do this, we compared the estimated SSRT with the average antagonist latency in each block of $\sim 200$ trials. This analysis also revealed strong positive correlation between these two measures $\left(r=0.70, p<10^{-8}\right.$ for the regression in Fig. 1C). More importantly, this block-by-block comparison permitted an analysis of cofluctuations in these two measures through time. If the antagonist latency is a valid proxy for movement cancellation, then block-by-block increases (decreases) in the SSRT should be associated with block-by-block increases (decreases) in the antagonist latency; as shown in Figure $1 D$ this was indeed the case $\left(r=0.61, p<10^{-8}\right.$ for the regression in Fig. $1 D$, with points distributing more often in the top right and bottom left quadrants than expected by chance; $\chi^{2}$ test, $\chi^{2}=$ $3.8, p=0.05)$. As a final analysis, for each subject in Experiments 2 and 3 (since these had six blocks), we ranked the blocks in order of lowest to highest SSRT and antagonist latency, and found that blocks with the shortest (longest) SSRT tended to also be those blocks with the shortest (longest) antagonist latency. To do this analysis, we took the difference in the rankings between SSRT and antagonist latency for each block and compared it to the average reshuffled ranking difference repeated 500 times. This was re- 

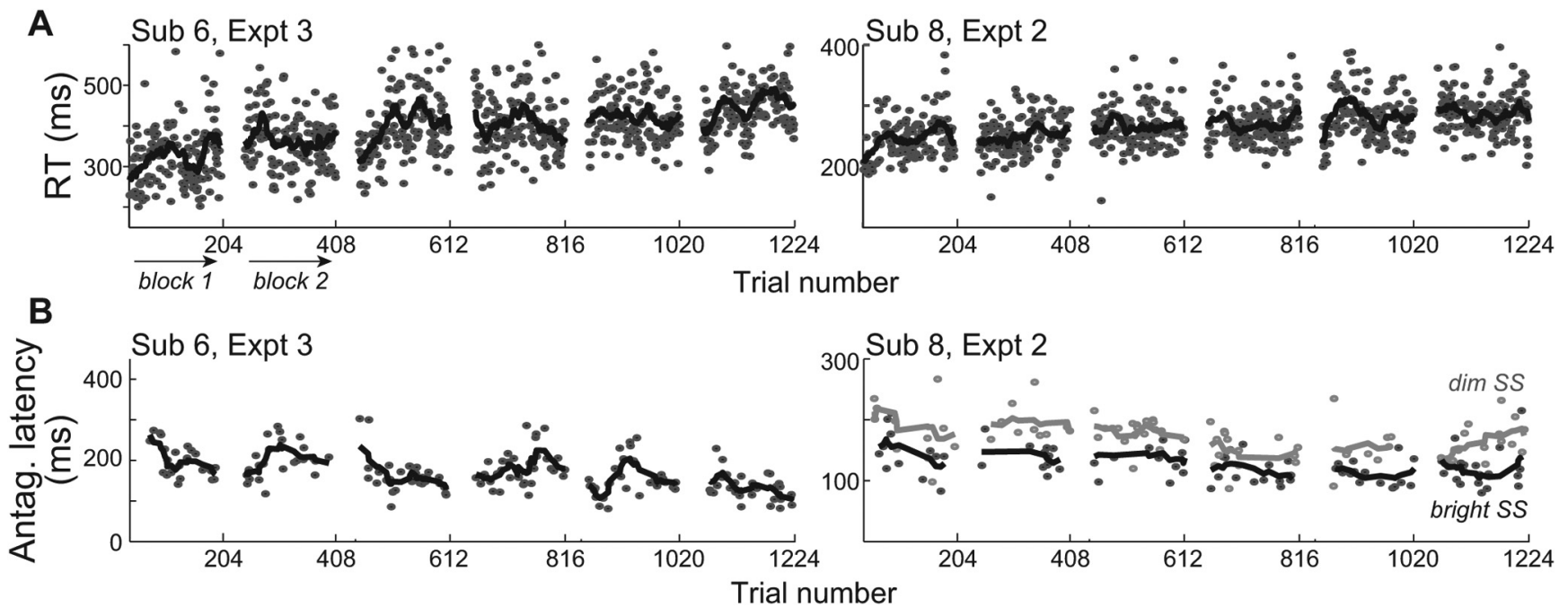

Figure 2. $\quad \boldsymbol{A}, \boldsymbol{B}$, Depiction of how $\mathrm{RT}(\boldsymbol{A})$ and antagonist latencies $(\boldsymbol{B})$ change within a block of 204 trials and across all six blocks for two representative subjects. Each circle shows an individual observation, and the lines show a running average ( 7 trials for RTs, 21 trials for antagonist latencies). Asterisks denote blocks where a linear trend within that block of trials reached significance. Different shading on symbols in $\boldsymbol{B}$ (right) represents the antagonist latency measure in response to different stop-signal intensities.

peated separately for dim and bright stop signals in Experiment 2, and the actual difference in rankings lay well below that predicted by the reshuffling (paired $t$ test, $t_{(25)}=-6.3, p<10^{-5}$ ). Together, all of these results are consistent with our hypothesis that the antagonist latency is a proxy for oculomotor cancellation.

\section{Nonstationarity and nonindependence of RTs and antagonist latencies}

Having established the close relationship between SSRT estimates of movement cancellation and the antagonist latency, we now investigate how antagonist latencies change through time. Previous work by Nelson et al. (2010) demonstrated that RTs on no-stop trials fluctuate on a variety of different short (i.e., previous-trial effects) and longer time scales. Are antagonist latencies prone to similar fluctuations, and, if so, how do such fluctuations relate to contemporaneous fluctuations in RTs? We therefore replicated the analyses of Nelson et al. (2010) on the RTs on no-stop-signal trials and extended them to the antagonist latencies from head-only errors (note that RT in our context refers to the onset of the high-velocity saccadic gaze shift unless noted otherwise).

We begin by showing how RTs on no-stop trials change through time, showing data from one representative subject in Experiment 3 (Fig. 2A, left) and a different subject in Experiment 2 (Fig. 2A, right). Recall that subjects in Experiments 2 and 3 performed six blocks of 204 trials each, with short breaks in between (Materials and Methods); the $x$-axis in Figure $2 A$ is accordingly broken between each of these blocks. From Figure $2 A$, it is clear that there is both substantial trial-by-trial variability in nostop trial RTs, as well as medium-term (i.e., within a block) and long-term (across all blocks) trends as well. We first examined the medium- and longer-term trends via a linear trend analysis either within a block or across all trials. A linear correlation between RT and trial number within a block of trials reached significance in 15 of 132 blocks across all three experiments, with positive slopes in 13 of these 15 significant correlations (acceptance values are Bonferroni-adjusted for multiple comparisons). These slopes were also significantly skewed to positive values across our sample ( $t$ test, $\left.t_{(131)}=3.94, p<0.0001\right)$. A linear correlation in the RTs across all trials reached significance in 16 of our 26 data sets
(Bonferroni-corrected for multiple comparisons), with positive slopes in 11 of these 16 significant correlations. These slopes tended to positive values across our sample, approaching significance $\left(t\right.$ test, $\left.t_{(25)}=2.03, p=0.053\right)$. Thus, across all three experiments, RTs on no-stop trials tended to increase both within and across blocks of trials. Figure $2 B$ shows examples of how the antagonist latency changes through time for the same two subjects. [Recall two different stop signals were used in Experiment 2; these are represented with different colored symbols in Figure $2 B$ (right).] As with RTs, considerable short-, medium-, and longterm variability is apparent in both subjects. To simplify comparison of the data across the three experiments, we analyzed only how the antagonist latency following a bright stop signal changed through time. (We confirmed that similar results were obtained if we only analyzed antagonist latencies following dim stop signals.) Two subjects (one from Experiment 1, one from Experiment 3) did not generate enough head-only movements to permit an analysis within or across blocks of $\sim 200$ trials. (The rate at which both of these subjects generated head-only errors was $>3$ SDs from the rates of the other subjects performing in the same experiment.) From the remaining 24 subjects, a linear trend analysis of the change in antagonist latency through a block of trials never reached significance (acceptance values Bonferroniadjusted for multiple comparisons), perhaps due to the smaller number of head-only error trials, nor was there any significant skew in the tendency for the antagonist latency to increase or decrease across a block of trials ( $t$ test of slopes, $t_{(122)}=-0.42$, $p=0.67)$. However, a linear correlation of antagonist latency across all trials reached significance in 4 of 24 subjects (Bonferroni-corrected for multiple comparisons), with negative slopes observed in all 4 cases. These slopes tended to negative values across our sample, but did not reach significance ( $t$ test, $\left.t_{(24)}=-1.73, p=0.09\right)$.

\section{Anticorrelated medium- and long-term trends in RTs and antagonist latencies}

The preceding analyses emphasize that both RTs and antagonist latencies exhibit considerable fluctuations on a number of different time scales. We now examine whether there is any relationship between the medium- and long-term linear trends in RTs 

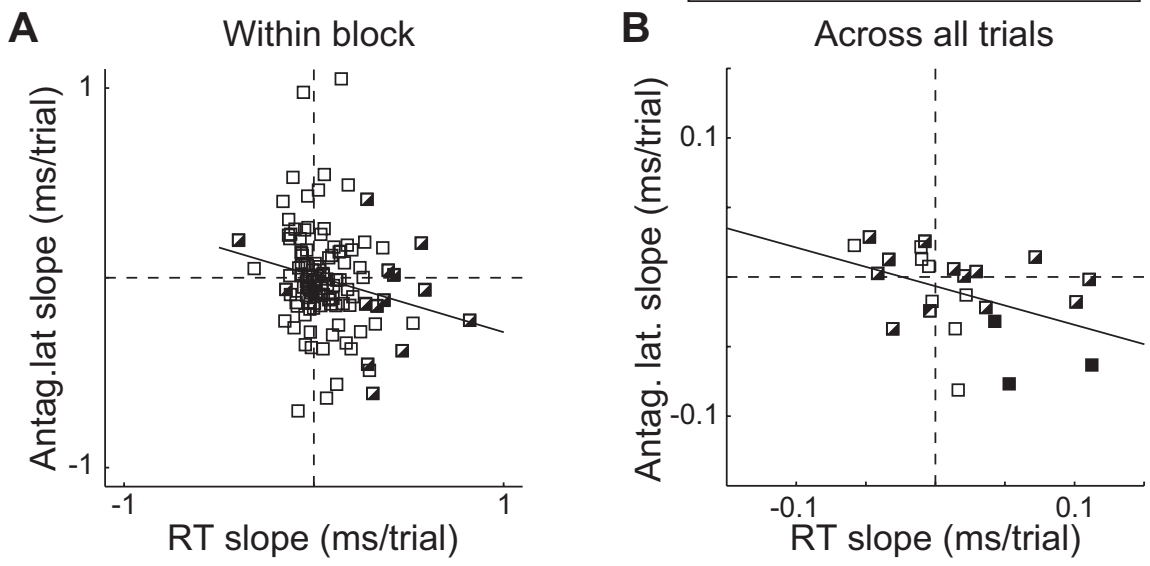

Figure 3. Linear trends in RTs or antagonist latencies. For each block of trials ( $\boldsymbol{A} ; 123$ squares total) or across all trials ( $\boldsymbol{B} ; 26$ squares total), we plotted the slope expressing the change in antagonist latency across trials as a function of the slope expressing the change in RTs across trials. Dashed lines divide quadrants; hence, values above or to the right of these lines represent measures that increased through time. Solid diagonal lines show a linear regression through these points $(p=0.039, A ; p=0.025, \boldsymbol{B})$, demonstrating that subjects that had larger increases in one measure through time tended to have larger decreases in the other measure.

and antagonist latencies observed within or across blocks, respectively, as this could provide insight into the nature of such fluctuations. For example, if the long-term changes in these measures were due to subject fatigue or dwindling motivation, then one would expect both measures to increase over time. Instead, a comparative analysis of the slopes of the changes in both measures either within a block of trials (Fig. $3 A$ ) or across all blocks (Fig. $3 B$ ) revealed a surprisingly anticorrelated trend: larger increases in one measure tended to be associated with larger decreases in the other measure $(r=-0.42, p=0.039$ for Fig. $3 A$; $r=-0.2, p=0.025$ for Fig. $3 B$ ). Furthermore, the data in both plots tended to cluster more in the top left or bottom right quadrants than would be expected by chance, approaching or exceeding significance $\left(\chi^{2}\right.$ test, $\chi^{2}=3.6, p=0.058$ for Fig. $3 A ; \chi^{2}=4.2$, $p=0.04$ for Fig. $3 B$ ), again emphasizing the anticorrelated nature of the trends in how RT and the antagonist latency change through time.

Another way of examining the same issue is to examine how the RT and antagonist latencies change within the same time window. To examine this, we performed a sliding-window analysis of the RT and antagonist latency averaged of over a 30 trial window and plotted the results against each other. (Nota bene, the 30 trial window length is arbitrary, and similar results were obtained with window lengths of $15,20,40,50$, or 100 trials; window lengths shorter than $\sim 15$ trials suffer from a lack of head-only errors.) Examples of these plots are shown in Figure 4, $A$ and $B$, for the data sets shown in Figure 2, $A$ and $B$, respectively. For both representative data sets, and regardless of the intensity of the stop signal in Experiment 2, we observed a general negative relationship; periods where RT averages tended to increase were associated with shorter antagonist latency averages and vice versa. Across our sample, the slopes of the regression relating these two averages together skewed significantly to negative values (Fig. $4 C$; $t$ test, $\left.t_{(34)}=-3.75, p<0.0001\right)$, and slopes did not differ depending on whether they were drawn from Experiments 1, 2 (bright stop signal only), or 3 ( $t$ tests, $p>0.39$ for all comparisons), or depending on whether a bright or dim stop signal was used (paired $t$ test, $t_{(7)}=-0.55, p=0.60$ ). Hence, the negative trend relating RT and antagonist latency within a subject did not depend on the intensity of the stop signal or whether the SSD was drawn randomly from a set of SSDs (Experiment 1) or determined in a staircasing fashion (Experiments 2 and 3 ).

\section{A triplet analysis of RTs and antagonist latencies}

We now turn to an examination of changes in the RT and antagonist latency on a trial-by-trial basis, as this may give insight into nature of short-term adaptive control of both movement generation and cancellation. The results up to now emphasize that both the RT and antagonist latency measures exhibit considerable fluctuations through time due to nonindependence and nonstationarity. As discussed by Nelson et al. (2010), these time-dependent fluctuations during the countermanding paradigm can confound the interpretation of any changes in the RT (and presumably antagonist latency) that may be occurring after a particular trial type. For example, since subjects go through intervals of responding sooner or later, which itself influences the probably of responding on a given stop-signal trial, any changes could be attributable to adaptive control, or may simply be a consequence of the nonstationarity and nonindependence of the measures. We therefore conducted a trial triplet analysis, as originally described by Nelson et al. (2010), since this analysis references the RT or antagonist latency measured after a given trial to the value before that trail, cancelling out the effects of nonstationary and nonindependent processes that act on longer time scales. In this analysis, the change in a parameter (shown for RTs on no-stop trials in Fig. 5A) across the first and third trials of a sequence is analyzed as a function of the type of the second, intervening trial. As applied for an analysis of RTs, note how trials $n-1$ and $n+1$ are no-stop trials for all sequences; the only difference between sequences $\mathrm{A}$ and $\mathrm{B}$ is the classification of trial $n$. Across our sample, RTs decreased significantly if trial $n$ was a no-stop trial (Fig. $5 A$, sequence A; paired $t$ test, $t_{(25)}=-8.5, p<10^{-8}$ ), and RTs increased if trial $n$ was a stop trial (Fig. $5 A$, sequence B; $t_{(25)}=4.4, p<0.001$ ). Similar results were observed with head RTs (results not shown). The patterns shown in Figure 5A largely replicate those reported previously by Nelson et al. (2010). There was no correlation between the size of these RT changes across no-stop versus stop trials on a subject-by-subject basis $(p=0.2)$.

We then performed a similar triplet analysis on the antagonist latency, examining changes in this value across trials $n-1$ and $n+1$ as a function of the type of trial on trial $n$. The number of appropriate trial sequences are far fewer, since the analysis requires head-only movements on trials $n-1$ and $n+1$. Since head-only errors were more likely in Experiments 2 and 3 given the use of a staircasing method to determine SSD, the number of appropriate sequences was greater in these experiments (recall as well that we only collected 600 trials total per subject in Experiment 1). We further restricted the data from Experiment 2 to those triplets where the same intensity stop signal was presented on trial $n-1$ and $n+1$. As a consequence of these constraints, 

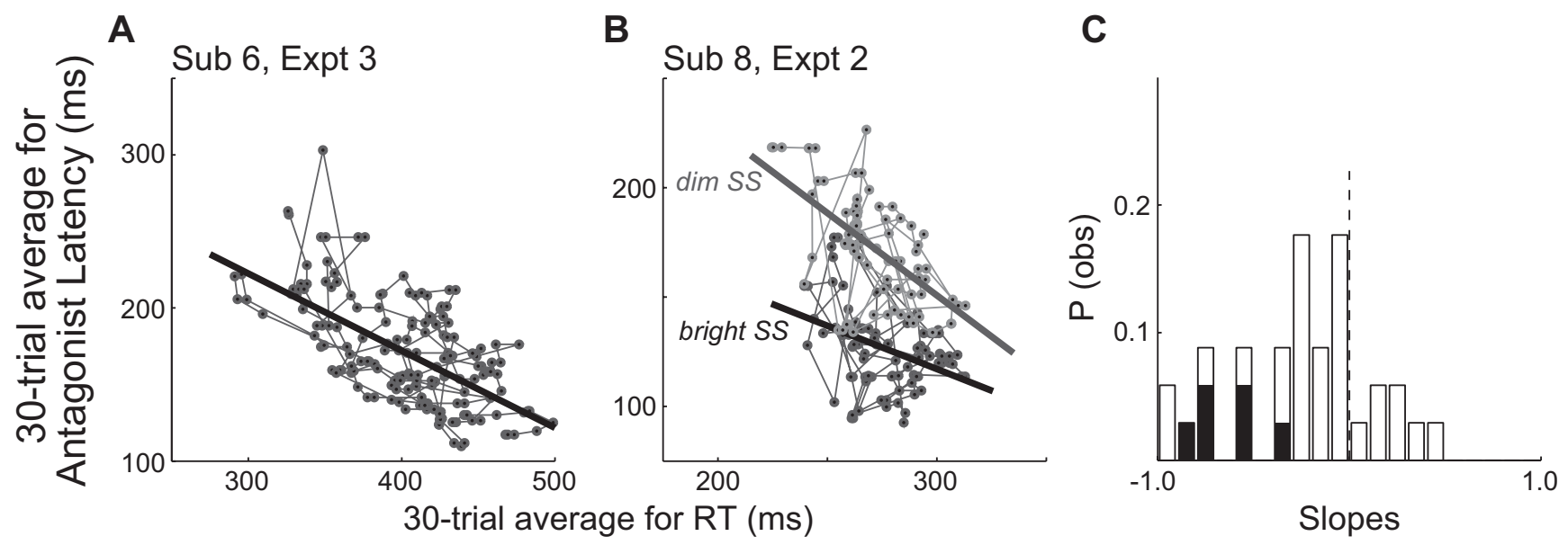

Figure 4. Sliding-window analysis of RT and antagonist latency. For each head-only error, we calculated the average RT and average antagonist latency for the 30 trials centered on the trial in question and plotted these averages against each other. $\boldsymbol{A}, \boldsymbol{B}$, Representative plots of this analysis for the subjects shown in Figure 2 . Each point represents an average, with thin lines connecting adjacent observations. Thicker solid lines show the result of a linear regression through the points. Different shaded symbols in $\boldsymbol{B}$ represent data derived from antagonist latencies in response to different intensity stop signals. C, Representation of regression slopes obtained from this analysis, pooled across our sample. Solid bins represent significant observations (Bonferroni-corrected for multiple comparisons).

subjects generated, on average, 3 such sequences in Experiment 1 (mean $\pm \mathrm{SD}, 2.6 \pm 1.7$; range, $1-6), 5$ such sequences in Experiment 2 (mean $\pm \mathrm{SD}, 4.6 \pm 1.6$; range, $3-8$ ), and 11 such sequences in Experiment 3 (mean $\pm \mathrm{SD}, 11.4 \pm 6.6$; range, 3-25). Across all subjects in all experiments, we obtained 96 sequences were the intervening trial was a no-stop signal and 68 sequences where the intervening trial was a stop-signal trial. When averaged within each subject, antagonist latencies did not change significantly if trial $n$ was a no-stop trial (Fig. $5 B$, sequence $\mathrm{A} ; t_{(25)}=$ $0.12, p=0.13$ ), but decreased significantly if trial $n$ was a stop trial

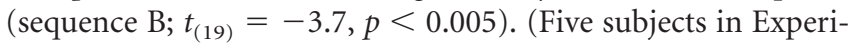
ment 1 did not have a sequence where trial $n$ was a stop trial.) There was also no correlation between the size of these changes in antagonist latency across no-stop versus stop trials on a subjectby-subject basis $(p=0.24)$. Importantly, although the antagonist latency decreases across stop trials, the RT of the head-only error actually increased across stop trials $\left(t_{(19)}=2.54, p=0.01\right)$, similar to the RTs shown in Figure $5 A$. This result confirms a fundamental difference between the initiation of the headonly error, which exhibits the same tendencies across trials as $\mathrm{RT}$, and the timing of antagonist muscle recruitment relative to the stop signal.

\section{Differing tendencies for short-term strategic adjustments in RTs and antagonist latencies}

We sought to further compare the changes in both RT and antagonist latency as a function of intervening trial type, performing two additional analyses that compared the subject-by-subject changes in RT and antagonist latency. In the first analysis, presented in Figure 5C, we show how either RT (squares) or antagonist latency (circles) changes across either intervening stop versus no-stop trials. Note from this plot how the changes in RT cluster in the top-left corner, meaning that RTs tended to decrease across no-stop trials and increase across stop trials. Such clustering in the top-right quadrant was highly significant $\left(\chi^{2}=\right.$ 39.5, $p<10^{-8}$ ). In contrast, the antagonist latency (Fig. $5 C$, circles) changed in a very different fashion across stop and nostop trials, clustering instead in the bottom left and bottom right quadrants. The difference between this distribution and one evenly distributed across all four quadrants approached signifi- cance $\left(\chi^{2}=7.6, p=0.055\right)$. Critically, the two distributions also differed significantly from each other in multiple ways, both in terms of the two-dimensional segregation of the circles and squares in Figure $5 C$ (2D Kolmogorov-Smirnov test, $d=0.52$; $p<0.01$ ), and the comparative changes in RT and antagonist latency across either no-stop trials (paired $t$ test, $t_{(19)}=-2.44$, $p=0.048$ ) or stop trials (paired $t$ test, $t_{(19)}=5.14, p<10^{-4}$; acceptance values are Bonferroni-adjusted for multiple comparisons).

Another way of representing the differences in how RTs and antagonist latencies change across different trial sequences is to plot how these values change against each other across either intervening no-stop trials (Fig. 5D, squares) or intervening stop trials (circles). Note again how the representations of the data in this manner cluster differently depending on trial sequences $(2 \mathrm{D}$ Kolmogorov-Smirnov test, $d=0.87 ; p<10^{-6}$ ), with changes across no-stop trials clustering in the top left and bottom left quadrants $\left(\chi^{2}=17.2, p<0.001\right)$, and changes across stop trials clustering in the lower right quadrant $\left(\chi^{2}=22, p<10^{-6}\right.$; acceptance values Bonferroni-adjusted for multiple comparisons).

Together, the analyses shown in Figure 5, $C$ and $D$, reinforce the differences in how the RT and the antagonist latency are adjusted across different trial sequences. Furthermore, the positioning of the various distributions in mostly mirroring quadrants emphasizes the opposing nature of adjustments to RTs and antagonist latencies: RTs tended to increase and antagonist latencies decrease across intervening stop trials (sequence B), but RTs tended to decrease and antagonist latencies increase (albeit not significantly) across intervening no-stop trials (sequence A).

Up to now, we have not subdivided our triplet analysis on stop trials based on whether subjects successfully cancelled a gaze shift or not, due to the small yield of triplets of head-only errors from which the antagonist latency could be extracted on a subject-bysubject basis. We therefore conducted another analysis where we first calculated the change in either the RT or antagonist latency across a given trial sequence and then pooled these changes across all triplets and subjects (differing from the within-subject analyses conducted on the data shown in Fig. 5). As shown in Figure $6 \mathrm{~A}$, RTs on no-stop trials tended to decrease with intervening no-stop trials (upward histogram), and tended to increase with 
A

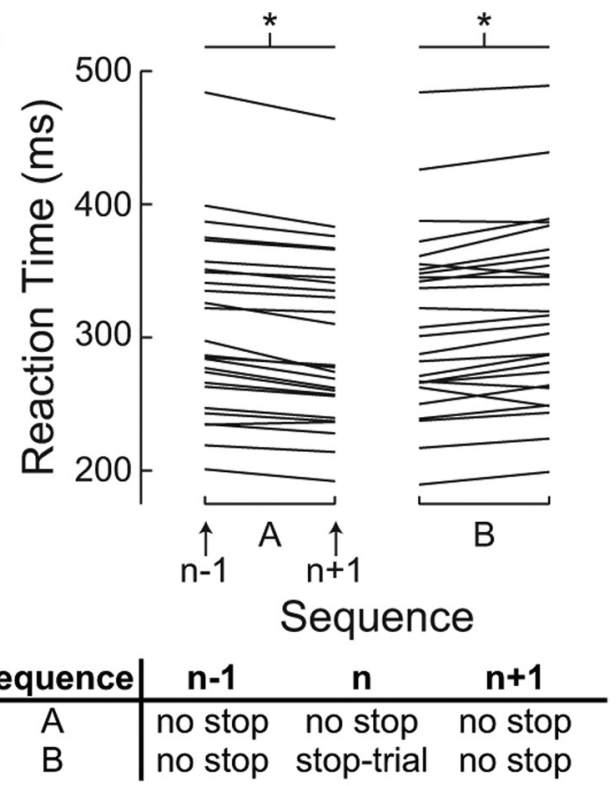

C

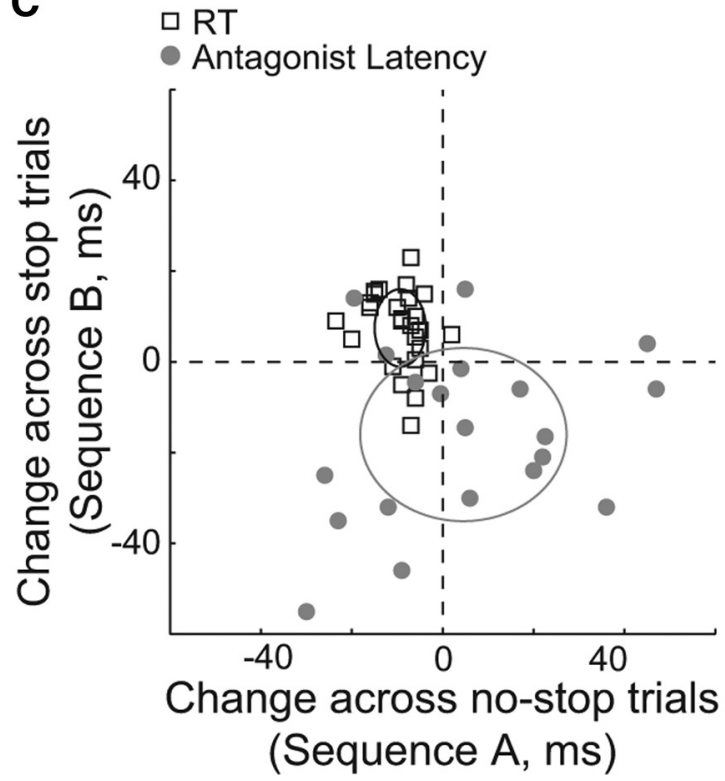

B
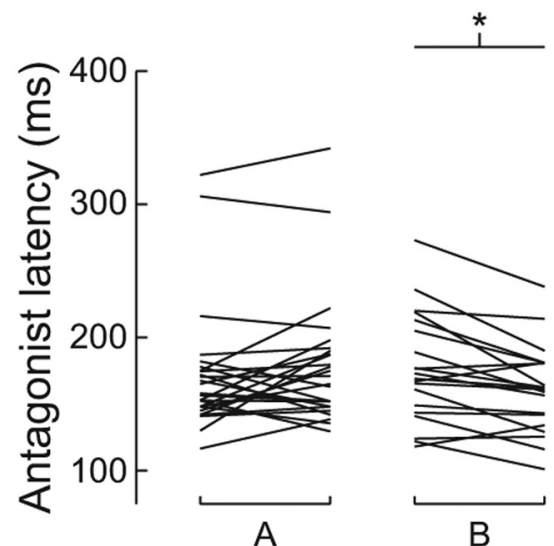

Sequence

\begin{tabular}{c|ccc} 
Sequence & $\mathbf{n}-\mathbf{1}$ & $\mathbf{n}$ & $\mathbf{n + 1}$ \\
\hline A & head-only & no stop & head-only \\
B & head-only & stop-trial & head-only
\end{tabular}

D

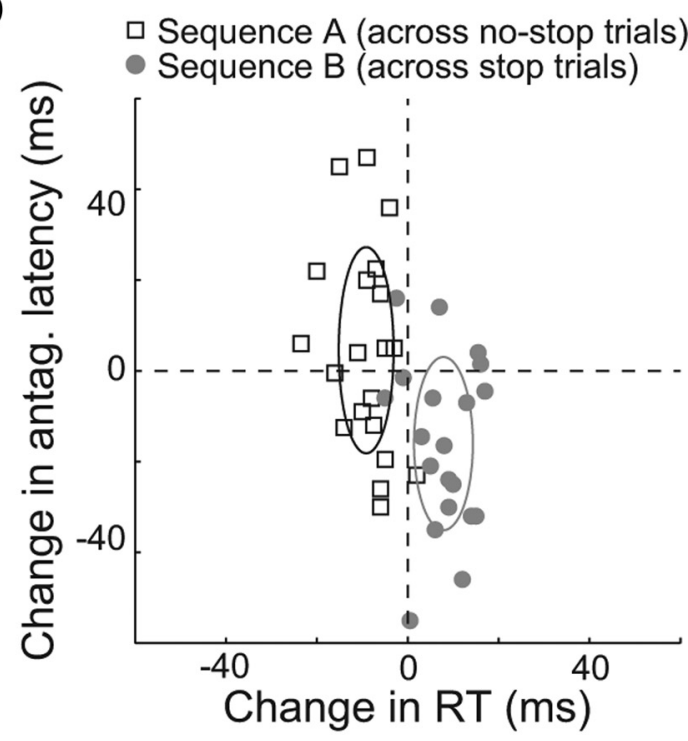

Figure 5. Triplet analysis of RTs and antagonist latencies. $\boldsymbol{A}, \boldsymbol{B}$, We analyzed either the change in no-stop signal RTs $(\boldsymbol{A})$ or antagonist latency $(\boldsymbol{B})$ as a function of intervening trial type (see legend below each plot for different sequences). Each line shows data from a different subject, and asterisks show significant differences from a paired $t$ test across the sample. $C$, Plot contrasting the change in sequence B (across stop trials) versus sequence A (across no-stop trials) for RT (squares) and the antagonist latency (circles). D, Plot contrasting the change in antagonist latency versus RT for sequence $A$ (across no-stop trials; squares) and sequence B (across stop trials; circles). Each symbol in $\boldsymbol{C}$ and $\boldsymbol{D}$ shows data from a different subject. Ellipses in $\boldsymbol{C}$ and $\boldsymbol{D}$ are centered on the mean of the data, with radii representing 1 SD.

intervening stop trials (downward histogram). Both distributions differed significantly from zero, given the very high number of observations (intervening no-stop trial, $-9.7 \pm 54.6 \mathrm{~ms} ; t$ test versus zero, $t_{(7624)}=-15.4, p<10^{-5}$; intervening stop trial, $\left.9.1 \pm 57.0 \mathrm{~ms}, t_{(3168)}=8.9, p<10^{-5}\right)$, and from each other (two-way $t$ test, $t_{(10791)}=-16.1, p<10^{-5}$ ). In contrast, as shown in Figure $6 B$, antagonist latencies tended to increase across an intervening no-stop trial (upward histogram; $6.4 \pm 38.0 \mathrm{~ms}$ ), although this distribution did not differ from zero $\left(t_{(95)}=1.6\right.$, $p=0.10)$. More importantly, antagonist latencies decreased significantly across an intervening stop trial (Fig. 6B, downward histogram; $\left.-15.6 \pm 41.7 \mathrm{~ms} ; t_{(67)}=-1.6, p<0.005\right)$, even though the head RT of head-only movements from the same trials actually increased (results not shown; $32.2 \pm 66.1 \mathrm{~ms}$; $\left.t_{(67)}=4.0, p<0.001\right)$. The distributions of antagonist latencies across stop versus no-stop trials also differed significantly from each other (two-way $t$ test, $t_{(162)}=3.5, p<0.001$ ). Furthermore, the change in RT and antagonist latencies differed significantly across both no-stop trials (Fig. 6, compare upward histograms; two-way $t$ test, $t_{(7719)}=2.9, p<0.005$ ) and across stop trials (Fig. 6 , compare downward histograms; two-way $t$ test, $t_{(3234)}=-3.5$, $p<0.001$; acceptance values are Bonferroni-adjusted for multiple comparisons).

Recall from our methods for Experiments 2 and 3 that we adaptively adjusted the SSD for a given stop trial based on the subject's behavior on the preceding stop trial, increasing or de- 


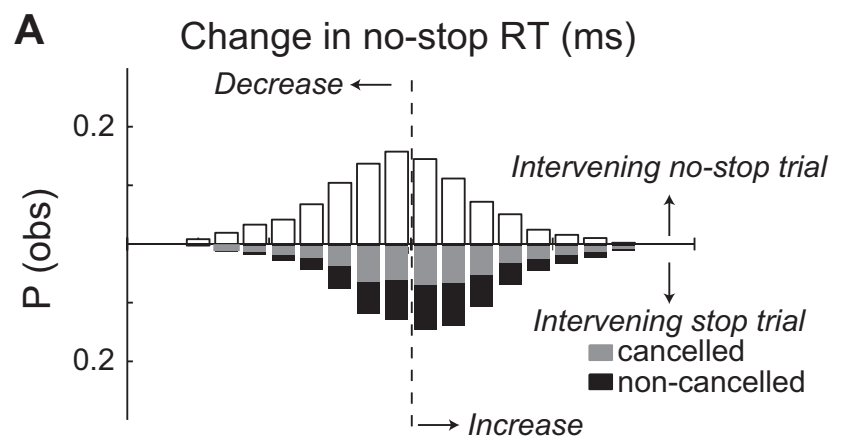

B Change in antagonist latency

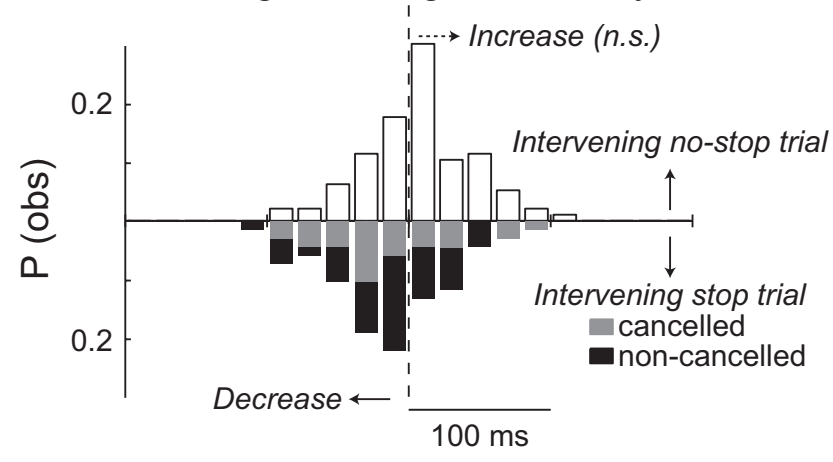

Figure 6. $\boldsymbol{A}, \boldsymbol{B}$, Frequency histograms of changes in either RT $(\boldsymbol{A})$ or antagonist latency $(\boldsymbol{B})$, collapsed across all subjects and segregated by different trial types. Upward empty histograms show how these measures change with an intervening no-stop-signal trial $(\boldsymbol{A}, 7625$ observations; $\boldsymbol{B}, 96$ observations); downward-filled histograms show how these measures change with an intervening stop-signal trial ( $\boldsymbol{A}, 3168$ observations; $\boldsymbol{B}, 68$ observations). Distributions that are significantly different from zero are indicated above/below the distributions with a solid arrow. The upward and downward distributions in both $\boldsymbol{A}$ and $\boldsymbol{B}$ are also significantly different from each other, emphasizing the differential influence of intervening trial type on RTs and antagonist latencies. Intervening stop trials are further subdivided into cancelled (gray bins) or noncancelled (black bins) trials.

creasing the SSD if the previous stop trial was cancelled or noncancelled, respectively. Given that we have demonstrated previously that antagonist latencies decrease for longer SSDs (Goonetilleke et al., 2010, 2012), could the results shown in Figure $6 B$ be a straightforward consequence of having longer SSDs, and hence shorter antagonist latencies, following cancelled stop trials? If so, we would expect that the antagonist latencies would decrease more across cancelled versus noncancelled stop trials, since SSDs increase after cancelled stop trials. We therefore subdivided the downward histogram in Figure $6 B$ by subject performance on the intervening stop trials. Contrary to what a simple relationship with SSD would have predicted, we found no difference in the decreases in antagonist latencies across cancelled or noncancelled stop trials (two-way $t$ test, $t_{(66)}=0.43, p=0.67$ ), regardless of whether or not we restricted these data to triplets obtained only from Experiments 2 and 3. A similar analysis on the change in RT showed slightly larger RT increases across sequences of noncancelled trials versus cancelled trials, approaching significance (two-way $t$ test, $t_{(3216)}=-2.2, p=0.06$ ). This final analysis demonstrates that the changes in antagonist latencies across stop trials are not simply a consequence of our method of adjusting the SSD, and also shows that the changes in both antagonist latency and RT across stop trials are similar regardless of subject performance.

\section{Discussion}

Using a countermanding paradigm, we have shown that the timing of a neuromuscular proxy of oculomotor cancellation obtained when the head is unrestrained fluctuates on a variety of time scales. This proxy is availably on a single trial, providing insights into the dynamic fluctuations of movement cancellation at an unprecedented resolution. Such fluctuations were largely the mirror opposite of those occurring contemporaneously on movement generation. These results advance the understanding of how processes dictating movement cancellation and generation are balanced in this task by demonstrating that both can be dynamically adjusted in similar, albeit opposing, manners.

\section{Comparison to previous results}

The SSRT remains a central metric for estimating the timing of movement cancellation (for review, see Verbruggen and Logan, 2008, 2009a; for an alternative, see Salinas and Stanford, 2013). Saccadic or manual SSRTs are not fixed for a given subject, but can vary, for example, depending on the fixation condition, the intensity or probability of the stop signal, or task instruction (Logan and Burkell, 1986; Morein-Zamir and Kingstone, 2006; Emeric et al., 2007; Morein-Zamir et al., 2007; Stevenson et al., 2009; Verbruggen and Logan, 2009b; Goonetilleke et al., 2012). SSRTs estimated through conventional means decrease following stop trials, and hence can also be adaptively adjusted (Bissett and Logan, 2012). Our results extend upon the notion of adaptive control of movement cancellation by directly demonstrating fluctuations within and across blocks of trials, and with immediate trial history. Moreover, our results emphasize an aspect of subject specificity, in that different subjects had different RT trends though time. What is remarkable, and inconsistent with explanations of fatigue or waning motivation, is how well such RT trends related to oppositely directed trends in the antagonist latency.

The influence of trial history on movement generation is well recognized in a variety of paradigms; RTs on trial $n$ are usually prolonged when trial $n-1$ involved a degree of conflict or an error (for review, see Fecteau and Munoz, 2003). Although many studies show that saccadic RTs are shorter when preceded by a no-stop versus a stop trial, RT increases are not seen consistently after noncancelled stop trials (Cabel et al., 2000; Kornylo et al., 2003; Ozyurt et al., 2003; Emeric et al., 2007), contrary to the notion of post-error slowing (Rabbitt, 1966). Nelson et al. (2010) reconciled this discrepancy by controlling for substantial fluctuations in RTs through time via a triplet analysis of the changes in $\mathrm{RT}$ across different types of intervening trials. Our results of a triplet analysis on RTs (Figs. 5A, 6A) replicate the findings of Nelson et al. (2010); RTs increased across intervening stop trials regardless of behavior, and decreased across no-stop trials.

Because the antagonist latency is available on a single trial, we extended the logic of the triplet analysis to movement cancellation. This analysis required head-only movements on trials $n-1$ and $n+1$, and hence the number of appropriate sequences is low. Despite this, we observed a consistent decrease in the antagonist latency across intervening stop trials that was not present across intervening no-stop trials (Fig. $5 B$ ), and indeed went in the opposite direction of the trends in RTs across the same sequence (Fig. 5A), as did the RT of the head on those same head-only movement trials. We also pooled the changes in antagonist latencies through various sequences across all subjects (Fig. 6) and observed a negligible effect of subject performance on the decrease in antagonist latency across stop trials. 
Perhaps the most revealing aspect of the triplet analyses is the comparison of the changes in RTs and antagonist latencies (Fig. $5 C, D)$. Across intervening stop trials, RTs increased, and antagonist latencies decreased; across intervening no-stop trials, RTs decreased, and antagonist latencies increased. These patterns, which were all $\sim 10 \mathrm{~ms}$ in magnitude, emphasize again the specific nature of adaptive control in this task with trial sequence. While a reasonable strategy following a stop trial would have been to slow all processes (i.e., shift a speed-accuracy trade-off to promote increased accuracy), our evidence favors an alternative interpretation whereby movement cancellation is selectively expedited while movement generation is slowed across stop trials. An opposing dynamic adjustment is made across intervening nostop trials, expediting movement generation while slowing movement cancellation.

\section{Is the antagonist latency an appropriate proxy of oculomotor cancellation?}

The interpretation of our results hinge critically on whether the antagonist latency is an appropriate proxy of oculomotor cancellation. We have considered this question more thoroughly previously (Goonetilleke et al., 2010, 2012), and new results presented here further support our contention. The antagonist latency correlates positively with the SSRT both within and across subjects and within and across blocks of trials (Fig. $2 A, B$ ), and decreases with increasing SSD (Goonetilleke et al., 2010; 2012), as expected of a proxy of movement cancellation since longer SSDs provide less time for stopping. Furthermore, as shown in Figure $2 C$, the block-by-block changes in SSRTs estimated by traditional means correlate positively with block-by-block changes in the antagonist latency, and blocks with the longest SSRT tend to be those with the longest antagonist latency.

More fundamentally, the appropriateness of the antagonist latency as a proxy for oculomotor cancellation depends on the nature of eye-head coordination during large saccadic gaze shifts. The circuits governing the initiation of orienting head movements are more permissive than those governing gaze shifts (for review, see Corneil, 2011); head movements can be both initiated and actively stopped even though gaze remains stable. Head-only movements can be considered as a partial response of the saccadic system (Corneil and Munoz, 1999; Corneil and Elsley, 2005), paralleling small muscle twitches, finger movements, or changes in force production that are occasionally made during otherwise successfully cancelled manual movements (Osman et al., 1986; De Jong et al., 1990; McGarry and Franks, 1997, 2003; McGarry et al., 2000; van Boxtel et al., 2001; Scangos and Stuphorn, 2010; Ko et al., 2012). Considering head-only movements as a type of partial response that are arrested midflight does not lessen the significance of our findings, so long as the initiation of antagonist recruitment arises from the processing of the stop signal. The selective recruitment of antagonist neck muscles on head-only movements, but not on fully cancelled or noncancelled eye-head gaze shifts that attain the target, supports this contention. The antagonist latency clearly fluctuates across a variety of time scales in a manner largely mirroring that occurring contemporaneously with RTs. A parsimonious explanation of these results is an adaptive and opposing adjustment of the priority given to movement generation and cancellation, as suggested previously (Bissett and Logan, 2011).

Oculomotor cancellation as an active, highly dynamic process The neurophysiology underlying antagonist muscle recruitment remains unclear. Neural correlates of oculomotor cancellation have been found in the decrease in movement-related activity and increase in fixation-related activity in the SC (Paré and Hanes, 2003) and FEF (Hanes et al., 1998). The decrease in movement-related activity within the oculomotor system is a common feature across a number of previous modeling studies of behavior in saccadic countermanding tasks when the head is restrained (Boucher et al., 2007; Lo et al., 2009; Salinas and Stanford, 2013). We do not believe it likely that either a decrease in movement-related activity or an increase in fixation-related activity could culminate directly in antagonist muscle recruitment. Subthreshold movement-related activity in the SC and FEF initiates activity on the agonist neck musculature (Rezvani and Corneil, 2008; Corneil et al., 2010); hence, withdrawal of movement-related activity would decrease agonist muscle activity, but not recruit antagonist muscles. Electrical stimulation in the rostral SC or lateral FEF produces little if any neck muscle recruitment, and never recruits anything like a braking pulse during ongoing motion (Corneil et al., 2002; Elsley et al., 2007). Electrical stimulation of omnipause neurons also fails to decelerate or prevent head motion despite robustly arresting or preventing gaze shifts (Gandhi and Sparks, 2007), further suggesting that an increase in fixation-related activity is not the proximate cause of antagonist muscle recruitment.

Instead, we speculate that detection of a stop signal, perhaps within frontostriatal circuits (for review, see Aron, 2011), initiates a parallel cascade of events that both shuts down the preparation of a developing oculomotor movement in the SC and FEF, and arrests any ongoing head motion through other circuits that are not yet fully understood. The expression of an active braking pulse on antagonist neck musculature before the initiation of a gaze shift suggests that oculomotor cancellation is not simply manifest as a withdrawal of an impending gaze shift. Our results illustrate the dynamics of such active braking, with cancellation being primed on precisely those trials where movement generation processes are being slowed. Whether such priming is accomplished via top-down presetting of the fixation system (with still unclear links to the cephalomotor system) (Lo et al., 2009) or expedited detection of the stop signal or expression of an inhibitory surge (Salinas and Stanford, 2013) remains to be determined.

\section{References}

Aron AR (2011) From reactive to proactive and selective control: developing a richer model for stopping inappropriate responses. Biol Psychiatry 69:e55-e68. CrossRef Medline

Bissett PG, Logan GD (2011) Balancing cognitive demands: control adjustments in the stop-signal paradigm. J Exp Psychol Learn Mem Cogn 37: 392-404. CrossRef Medline

Bissett PG, Logan GD (2012) Post-stop-signal adjustments: Inhibition improves subsequent inhibition. J Exp Psychol Learn Mem Cogn 38:955966. CrossRef Medline

Boucher L, Palmeri TJ, Logan GD, Schall JD (2007) Inhibitory control in mind and brain: an interactive race model of countermanding saccades. Psychol Rev 114:376-397. CrossRef Medline

Cabel DW, Armstrong IT, Reingold E, Munoz DP (2000) Control of saccade initiation in a countermanding task using visual and auditory stop signals. Exp Brain Res 133:431-441. CrossRef Medline

Corneil BD (2011) Eye-head gaze shifts. In: The oxford handbook of eye movements (Liversedge SP, Gilchrist ID, Everling S, eds), pp 303-322. Oxford, UK: Oxford UP.

Corneil BD, Elsley JK (2005) Countermanding eye-head gaze shifts in humans: marching orders are delivered to the head first. J Neurophysiol 94:883-895. CrossRef Medline

Corneil BD, Munoz DP (1999) Human eye-head gaze shifts in a distractor task. II. Reduced threshold for initiation of early head movements. J Neurophysiol 82:1406-1421. Medline 
Corneil BD, Hing CA, Bautista DV, Munoz DP (1999) Human eye-head gaze shifts in a distractor task. I. Truncated gaze shifts. J Neurophysiol 82:1390-1405. Medline

Corneil BD, Olivier E, Munoz DP (2002) Neck muscle responses to stimulation of monkey superior colliculus. I. Topography and manipulation of stimulation parameters. J Neurophysiol 88:1980-1999. Medline

Corneil BD, Elsley JK, Nagy B, Cushing SL (2010) Motor output evoked by subsaccadic stimulation of primate frontal eye fields. Proc Natl Acad Sci U S A 107:6070-6075. CrossRef Medline

De Jong R, Coles MG, Logan GD, Gratton G (1990) In search of the point of no return: the control of response processes. J Exp Psychol Hum Percept Perform 16:164-182. CrossRef Medline

Elsley JK, Nagy B, Cushing SL, Corneil BD (2007) Widespread presaccadic recruitment of neck muscles by stimulation of the primate frontal eye fields. J Neurophysiol 98:1333-1354. CrossRef Medline

Emeric EE, Brown JW, Boucher L, Carpenter RH, Hanes DP, Harris R, Logan GD, Mashru RN, Paré M, Pouget P, Stuphorn V, Taylor TL, Schall JD (2007) Influence of history on saccade countermanding performance in humans and macaque monkeys. Vision Res 47:35-49. CrossRef Medline

Fecteau JH, Munoz DP (2003) Exploring the consequences of the previous trial. Nat Rev Neurosci 4:435-443. CrossRef Medline

Gandhi NJ, Sparks DL (2007) Dissociation of eye and head components of gaze shifts by stimulation of the omnipause neuron region. J Neurophysiol 98:360-373. CrossRef Medline

Goonetilleke SC, Doherty TJ, Corneil BD (2010) A within-trial measure of the stop signal reaction time in a head-unrestrained oculomotor countermanding task. J Neurophysiol 104:3677-3690. CrossRef Medline

Goonetilleke SC, Wong JP, Corneil BD (2012) Validation of a within-trial measure of the oculomotor stop process. J Neurophysiol 108:760-770. CrossRef Medline

Hanes DP, Patterson WF 2nd, Schall JD (1998) Role of frontal eye fields in countermanding saccades: visual, movement, and fixation activity. J Neurophysiol 79:817-834. Medline

Ko YT, Alsford T, Miller J (2012) Inhibitory effects on response force in the stop-signal paradigm. J Exp Psychol Hum Percept Perform 38:465-477. CrossRef Medline

Kornylo K, Dill N, Saenz M, Krauzlis RJ (2003) Cancelling of pursuit and saccadic eye movements in humans and monkeys. J Neurophysiol 89: 2984-2999. CrossRef Medline

Lo CC, Boucher L, Paré M, Schall JD, Wang XJ (2009) Proactive inhibitory control and attractor dynamics in countermanding action: a spiking neural circuit model. J Neurosci 29:9059-9071. CrossRef Medline

Logan GD, Burkell J (1986) Dependence and independence in responding to double stimulation: a comparison of stop, change, and dual-task paradigms. J Exp Psychol Hum Percept Perform 12:549-563. CrossRef

Logan GD, Cowan WB (1984) On the ability to inhibit thought and action: a theory of an act of control. Psychol Rev 91:295-327. CrossRef

McGarry T, Franks IM (1997) A horse race between independent processes: evidence for a phantom point of no return in preparation of a speeded motor response. J Exp Psychol Hum Percept Perform 23:1533-1542. CrossRef Medline

McGarry T, Franks IM (2003) On the nature of stopping an earlier intended voluntary action. Motor Control 7:155-198. Medline

McGarry T, Inglis JT, Franks IM (2000) Against a final ballistic process in the control of voluntary action: evidence using the Hoffmann reflex. Motor Control 4:469-485. Medline

Morein-Zamir S, Kingstone A (2006) Fixation offset and stop signal intensity effects on saccadic countermanding: a crossmodal investigation. Exp Brain Res 175:453-462. CrossRef Medline

Morein-Zamir S, Chua R, Franks I, Nagelkerke P, Kingstone A (2007) Predictability influences stopping and response control. J Exp Psychol Hum Percept Perform 33:149-162. CrossRef Medline

Nelson MJ, Boucher L, Logan GD, Palmeri TJ, Schall JD (2010) Nonindependent and nonstationary response times in stopping and stepping saccade tasks. Atten Percept Psychophys 72:1913-1929. CrossRef Medline

Osman A, Kornblum S, Meyer DE (1986) The point of no return in choice reaction time: controlled and ballistic stages of response preparation. J Exp Psychol Hum Percept Perform 12:243-258. CrossRef Medline

Ozyurt J, Colonius H, Arndt PA (2003) Countermanding saccades: evidence against independent processing of go and stop signals. Percept Psychophys 65:420-428. CrossRef Medline

Paré M, Hanes DP (2003) Controlled movement processing: superior colliculus activity associated with countermanded saccades. J Neurosci 23 : 6480-6489. Medline

Rabbitt PM (1966) Errors and error correction in choice-response tasks. J Exp Psychol 71:264-272. CrossRef Medline

Rezvani S, Corneil BD (2008) Recruitment of a head-turning synergy by low-frequency activity in the primate superior colliculus. J Neurophysiol 100:397-411. CrossRef Medline

Salinas E, Stanford TR (2013) The countermanding task revisited: fast stimulus detection is a key determinant of psychophysical performance. J Neurosci 33:5668-5685. CrossRef Medline

Scangos KW, Stuphorn V (2010) Medial frontal cortex motivates but does not control movement initiation in the countermanding task. J Neurosci 30:1968-1982. CrossRef Medline

Stevenson SA, Elsley JK, Corneil BD (2009) A "gap effect" on stop signal reaction times in a human saccadic countermanding task. J Neurophysiol 101:580-590. Medline

van Boxtel GJ, van der Molen MW, Jennings JR, Brunia CH (2001) A psychophysiological analysis of inhibitory motor control in the stop-signal paradigm. Biol Psychol 58:229-262. CrossRef Medline

Verbruggen F, Logan GD (2008) Response inhibition in the stop-signal paradigm. Trends Cogn Sci 12:418-424. CrossRef Medline

Verbruggen F, Logan GD (2009a) Models of response inhibition in the stopsignal and stop-change paradigms. Neurosci Biobehav Rev 33:647-661. CrossRef Medline

Verbruggen F, Logan GD (2009b) Automaticity of cognitive control: goal priming in response-inhibition paradigms. J Exp Psychol Learn Mem Cogn 35:1381-1388. CrossRef Medline 